last from ten to fifteen minutes, "seemed to be caused by the drawing of the breath." Sometimes the patient would hold his breath to keep from having the pains,

A year or two ago had twitching of the eyelids on the right side. At that time the numb skin on the trunk felt creepy, especially when the clothes would touch it, and the patient felt as though walking on sand all the time.

A year ago last fall his eye trouble became worse--eyes saw double, and this double vision lasted for about two or three weeks, could hardly see across the house, could see double each way, to the right and left; would not see double when looking straight forward. After looking to the right or left it would take a little while to get the eyes pointed straight forward again.

March, 1896. -No heterophoria. V. D. $=20 / 30 ;$ with $+.50 \mathrm{C}$. axis $90^{\circ}=2 \% / 20$. V. L. $=2 \% / 40 ;$ with +.50 C. axis $90^{\circ}=2 \%$, to which was added +3.50 for near vision. A test with the perimeter shows a slight narrowing of the field for red and a central scotoma in field of left eye. Patient no longer gets dizzy, except in bed at night, when he is awakened by a great noise in the head, and will be dizzy until he can assume a new position. The glottis does not completely close in spasm, though he occasionally has a tickling spasmodic cough of short duration.

December, 1896.-Does not strangle, and the cough is slight and controllable. Vocal cords during phonation are parallel. When getting his breath after having spoken a few words his inhalations are audible. Mucous membrane of the throat is pale. Vocal cords during phonation are not steady. The left vocal cord is in the median line and remains there during the use and non-use of the voice. During phonation the right vocal cord will come up in to position parallel with the left, and then by a series of unsteady movements will go away from its partner. During inhalation the left cord remains in the median line and is sucked downward like a valve, while the right vocal cord is curved outward, being sc mewhat sickleshaped, and is valve-like but not depressed as much as the left.

May 11, 1897.- The patient has had but one attack of strangling in several months, no dizziness, no pains, no difficulty in micturition or defecation, no hemorrhoids. The field of vision remains about the same. The scotoma in the right eye is not now demonstrable, and that in the left eye is much smaller, being but a mere line extending from the point of fixation half way across the nasal portion of the field.

\section{SALIVARY CALCULI.}

Presented to the Section on Laryngoloyy and Otology, at the Forty eighth Annual Meeting of the American Medical Association, held at Philadelphia, Pa., June 1-4, 1897.

BY W. FREUDENTHAL, M.D.

PRESIDENT OF THE GERMAN MEDICAL SOCIETY OF THE CITY OF NEW YORK. NEW YORK, N. Y.

Salivary calculi are, as a rule, easily recognized, although they are not of frequent occurrence. They are most frequently located near the buccal end of Wharton's or Steno's duct, and often have a definite outline and shape, rendering their detection by palpation usually easy. There are, however, a number of cases recorded in which their discovery was made by mere chance. Such an instance occurred in my practice. In two of my cases the diagnosis was easily made.

It appears that the growth and formation of salivary calculi begin years before their detection, and in this respect their character is quite in contrast to the stones found imbedded in the nasal cavities, which latter reach a large size after sometimes a growth of only a few months. The secretory function of Steno's or Wharton's duct and of the nasal cavity proper are not only different, but the foreign bodies found there are of different sizes. Sometimes these ducts contain large stones, without in any way producing symptoms, or in any sense annoying the patient. Such an instance is the following case:

Case 1.-Mr. A. F., 23 years of age, for the last six years a professional clarionetist, came to me last winter, in the height of his season, complaining of a cold, contracted two weeks before and in consequence of this an abscess had formed, as he be- lieved, under the tongue. On examination I found a swelling about the size of a hen's egg in the left sublingual region. No hard substance could be felt at all. Believing that I was deal ing with a ranula, I made a long incision through the buccal mucosa. A mass of fetid pus escaped, and, to my surprise, the end of a stone, oblong in shape, appeared in the opening. It was readily extracted. It undoubtedly came from Wharton's duct, judging from its contour. There is nothing unusual in its size and weight.

After thoroughly cleansing the abscess cavity, I passed a probe, and was surprised to find evidence of another stone situated deeply within the tissues of the submaxillary gland. My first effort to extract it resulted in failure, as it slipped back further in the cavity. My second attempt, however, was suc cessful. The stone was irregularly oval and about as large as a cherry. One would suppose that a person whose mouth is constantly in action by reason of his professional occupation, would be able to detect anything of an abnormal character in it. Yet in this instance the patient never suspected anything whatever, until two weeks ago, when as he states, he contracted the cold, which most likely caused the abscess about which he consulted me. $\mathrm{He}$ is positive that before contracting his cold he never felt anything unusual in his mouth.

As an illumination of the powers of endurance, it is interesting to state that my patient, a weak man at best, not only followed his usual vocation as a clario. net player up to the day on which the calculus was discovered and extracted, but also played his musical instrument on the evening after the operation despite the fact that there was a large iodoform tampon in the old site of the calculus. There are on record other instances where a supposed "cold" led to the detection of salivary calculi.

Mr. Carver ${ }^{1}$ removed a stone from a man 70 years of age, who had noticed some tenderness in the sublingual region only for about five or six weeks.

Demons ${ }^{2}$ found a stone the size of an olive in Wharton's duct, which produced no symptoms until three weeks before its removal.

There are, on the other hand, cases recorded in which the stone had made its presence felt for several years before its final extraction. Alfred Smith and Alex. T. Scott ${ }^{3}$ reported a case in which a tumor had been first noticed by the patient seven years before, at which time it was the size of a pea.

Jos. Dixon ${ }^{4}$ describes the sufferings of his wife, who had noticed a swelling under the tongue for fifteen years. Mr. Alexander Bruce ${ }^{5}$ removed a ston $\theta$ of "considerable dimensions," as he states it, although it weighed only $11 \frac{1}{2}$ grains. In this instance the patient had felt it fourteen years previously. Dr. Webb's ${ }^{6}$ patient felt a small lump sixteen years before being operated upon. Dr. Padieu's ${ }^{7}$ patient felt it for twenty-five years, and Dr. Schuster's ${ }^{8}$ patient for forty-four years (!)

It is known that patients suffering from salivary calculi frequently obtain relief from their symptoms by squeezing out the pus and mucus which surround the stone and fill the cavity, into the mouth, from which they expectorate it or unconsciously swallow it. Thus, we can understand in Jos. Dixon's case the attacks of dyspepsia lasting for fifteen years, which were not amenable to treatment. The patient apparently swallowed the secretions which she pressed out from the glands.

Other symptoms caused by the stones are pain increased by movements of the jaw such as during mastication, and from retention of saliva shortly after mas-

The Lancet, March 13. 1886, p. 494

Jour. de med. et de chir. de Bordeaux, Sept. 6, 1885

Jour. of the British Dental A ssoc., 1888, p. 175

Lancet, Nov. 26, 1887, p. 1063.

Transactions of the Pathol. Soc. of London, Vol. xvii, p. 184.

Ibidem, viii, p. 221.

Gazette Med., June 6, 1885. 
tication. Still another symptom occasionally encoun. tered is difficulty in breathing. That this last symptom is sometimes dangerous to life is proven by the case of J. W. Hulke, and my second case.

Hulke ${ }^{9}$ treated a young lady who had a tumor under the tongue which had been growing for the last six years. This tumor sometimes enlarged temporarily to such proportions as to threaten suffocation. At times there would be a discharge into the mouth, followed by the return of the swelling to its former size. The patient indicated by her statements that the stone had nearly suffocated her.

The following case which came under my observation, illustrates this last point:

Case 2.-Mr. N. S., aged 45 years, had been suffering two and a half years before he noticed a swelling under the tongue. This swelling grew steadily, and occasionally caused him great pain. For the last ten days the pain and swelling had grown worse. During the night he snored so loudly that he had to be wakened, and on these occasions his face would become markedly cyanotic. The patient was tall and very stout, and had the hoarse voice that characterizes the chronic alcoholic. On examination the tumor was found to project slightly from under the tongue and to extend along the right of the frenulum, reaching to the floor of the mouth, backward between the root of the tongue and the angle of the jaw, at which spot it appeared to be adherent to the buccal mucous membrane, simulating the case described by Hulke. By palpation fluctuation was elicited, and a hard mass could be plainly felt within it. I was quite sure that we had to deal here with a ealivary calcu. lus which had formed in the beginning of the main duct, and I advised its removal. For obvious reasons the patient refused and went home. His heart and lungs were normal.

Several weeks later I received a note from his physician, stating that the patient had died. The night preceding his death he had imbibed more freely than usual, and then retired. Having fallen asleep, he began to snore so loudly that his wife awoke. She found the patient breathing with great difficulty, while his face was black and blue. She tried to awaken him, but failed. She had a physician called; but the patient died of suffocation before medical aid reached him. An autopsy disclosed an almond-shaped salivary calculus, imbedded in a pus cavity. Death no doubt resulted indirectly from its presence; for on previous occasions he would invariably get rid of the pus that had accumulated in the cavity by coughing or hawking it out, and in this manner relieve himself of the cause of his dyspnea. But on the fatal night he was so fully under the influence of liquor that he could not arouse himself from the stupor caused by it, and thus succumbed.

I remarked in the beginning of this paper, that when I compared the sizes of salivary calculi and rhinoliths that I believed that the majority of these deposits owe their formation to the fact that a foreign body enters the duct or gland itself.

When Clinton Wagner ${ }^{10}$ says: "These concretions are formed by the deposit of earthy salts from the saliva in the excretory ducts leading from the gland or in the body of the gland itself, and that the cause of the deposit is an obstruction to the flow of saliva either to or through the excretory duct," he overlooks the primary cause of their formation, $i$. e., a foreign body. Around this foreign body is deposited layer after layer of earthy salts. This obstruction causes a retention of the salivary secretion. I do not, however, claim that this process obtains in all instances of salivary calculi, but in the majority.

Professor Ribbert of Bern, found that a hair of a

9 Transactions of the Pathol. Soc. of London, Vol. xxiv, p. 88.

10 N. Y. Med. Jour., Nov. 11, 1893, p. 560. toothbrush had forced itself into Wharton's duct, thus giving rise to the formation of a salivary calculus around it.

In several other instances the same causative ele. ment was found. Hulke removed a stone weighing 76 grains. Its broken surfaces exhibited concentric rings around a small, black. central speck. This speck was a fragment of wood.

It must be true that in certain calculi no central foreign body can be demonstrated, because it has undergone chemical changes. Galippe reported a case of spontaneous discharge of a stone from Wharton's duct. This calculus had concentric strata without a kernel being found. But he found microbes, already described by Malassez and Vignal, in these deposits.

One case is reported by Wertheimer ${ }^{11}$ in which an abscess formed during eating. The patient while eating blueberries suddenly noticed a tumor in the right submaxillary region, and at the same time a burning sensation in the mouth These symptoms always appeared during mastication. Finally, among greenish cheesy masses, the berries were found. Here stones had not yet formed, so, properly speaking this case does not belong here, but it shows how often foreign bodies enter Wharton's duct.

Case 3.-Mr. M. P., 57 years of age, merchant, of moderate habits and enjoying good health, had noticed for some time a small lump in the left submaxillary region. For the last three months it had grown steadily and had become painful during and after mastication. A simple incision was made, and a small oval shaped stone removed. In cutting it open, concentric rings were seen and a very small piece of wood in the center. It was ascertained that the patient had been for years in the habit of chewing toothpicks. thus most likely forcing the foreign body into Wharton's duct.

These cases certainly prove that the formation of calculi around a foreign body does occur in these as well as in other parts of the human anatomy. That there are other causes for their formation there is no doubt, an increase or decrease in the quantity of saliva is an important factor.

A case of Thomas Ballard ${ }^{12}$ is of interest: A young lady suffered from the effects of a buccal abscess which had opened spontaneously. Two small calculi were removed from the abscess cavity. It was learned that eleven years before she was salivated during an attack of jaundice. It is probable that this salivation caused a swelling of the excretory duct of the gland, then a retention of saliva and gradually the formation of a stone.

Just as increased flow of saliva may tend to produce a calculus, it may also tend to wash away a nucleus in the duct, around which salty deposits might form. This was the case with a patient of Kurz's, who had taken pilocarpin internally. The increased ylandular excretion washed out a calculus from Wharton's duct.

In conclusion, I also believe that the submaxillary is more often the site and seat of calculi than the parotid gland, because the secretion of the former gland contains a comparatively larger proportion of mucin, a substance which seems to possess considerable adhesive properties, and seems to favor the deposit of inorganic salts. This belief is confirmed by the fact that the usual site of the calculus is just at the outlet of the salivary duct on the buccal mucosa, a point which furnishes a ready ingress for foreign matter.

1003 Madison Ave.

11 Corps etrangers du canal de Wharton. Bulletin méd. du Nord. Dec. 12 Transactions of the Pathol. Soc. of London. Vol. xviii, p. 93. 
DISCUSSION.

Dr. Solly-I once found a calculus in the submaxillary gland. I have never seen u case reported where the calculus was imbedded in the body of the gland. An incision was made following up the duct and the stone was felt by the finger, imbedded in the gland, and was then forced into the mouth and removed. The stone was about the size of a chestnut and weighed twenty-five or thirty grains.

Dr. Freudenthal -..I am sure that there are several cases on record where the stone was in the body of the gland. Dr. Bull of New York has one, and there is a case reported by another surgeon whose name I do not recall, in which the stone could not be reached from the inside of the mouth and he had to make an incision from the outside.

\section{AN UNUSUAL CASE OF BLOOD CYST OF THE POSTERIOR NARES.}

Presented to the Section on Laryngology and Otology at the Forty-eighth Annual Meeting of the American Medical Association, held at Philadelphia, Pa., June 1-4, 1897.

BY JOHN N. MACKENZIE, M.D. NEW YORK, N. Y.

The patient was a young lady of 23 . As a result of the imperfect extraction of a tooth, there was necrosis of the jaw, with subsequent exfoliation of bone, leading to a more or less complete obliteration of the lower portion of the antrum. This had happened five years before consultation. For three years before consultation she had suffered with symptoms referable to the orbit and antrum. On examination a small glistening point was seen, resembling the appearance seen in fibroma of the nasal cavity. The right side of the nose was occluded. The mass looked very much like a child's toy balloon. The envelope of the cyst was extremely attenuated looking as if it was scarcely a line in depth. I made an appointment with her to return the same day, but in the meantime a spontaneous rupture occurred, and when I examined her the next day I was unable to find any trace of the growth. The inflammatory process which originated the trouble probably started in the antrum as a result of the necrosis of bone, and from this the edema and the subsequent formation of the cyst had resulted. I consider this a unique case. Some cases of large serous cysts have been reported.

AN ETIOLOGIC STUDY OF ATROPHIC DISEASE OF THE UPPER AIR PASSAGES BASED UPON AN EXAMINATION OF TWO HUNDRED CASES.

Presented to the Section on Laryngology and Otology at the Forty-eighth Annual Meeting of the American Medical Association held at Philadelphia, June 1-4, 189i.

BY J. L. GOODALE, M.D. BOSTON, MAsS.

In the following paper I desire to invite your attention rather to the predisposing than to the immediate causes of atrophic states of the nose, pharynx and larynx. The statements current in our recent textbooks that certain classes of persons under certain conditions are particularly liable to these conditions appear to be derived from general impressions. Since under such circumstances, a few positive examples are apt to outweigh unduly a large number of negative and therefore easily forgotten ones, I undertook recently to submit the current statements to the test of a systematic examination. For this purpose 200 cases of atrophic disease of the nose, pharynx or lar- ynx were investigated carefully with reference to the following points.

1. The relation of these states to age, sex, sexual functions in the female, and general nutrition.

2 . The influence of a normal patency of the anterior nares.

3. The influence of the inhalation of an insanitary atmosphere.

4. The influence of pre-existing local or general disease.

5 . The relation to associated hypertrophic states of the neighboring mucous membrane or lymphoid structures.

In the consideration of atrophic disease, I have at the outset excluded the following conditions:

1. An imperfect development of the intra-nasal structures, especially of the turbinates, occurring in chlorotic young women, and not associated with nasal obstruction or chronic inflammation of the nasal mucous membrane. For purposes of convenience, I have called this condition congenital hypoplasia of the nasal turbinate, since, although histologic examinations are wanting, an analogy is very directly suggested to Virchow's congenital hypoplasia of the female sexual and vascular systems associated with chlorosis.

2. I also exclude an imperfect development of the intra-nasal structures occurring in post-nasal obstruction, probably due as I have previously suggested ${ }^{1}$ to the absence of normal respiratory alterations in intranasal air pressure requisite for proper nutrition.

3. There are to be excluded the anemic conditions of the nasal mucous membrane, secondary to general malnutrition, particularly seen in phthisis; also

4. The pressure atrophies from septal deviations and new growths.

5. Finally, atrophic states associated with syphilitic lesions in the vicinity.

With the exception perhaps of the last, such states are the result of altered nutrition and non-inflammatory in nature. They are therefore sharply to be dis. tinguished from the genuine atrophy at present under consideration. The five conditions just referred to although recognizable when occurring typically may, if inflammation supervenes, closely resemble clinically mild forms of genuine atrophy. This possible source of error is to be borne in mind in the examination of cases.

For purposes of classification it appeared most natural to divide the cases of true atrophy into the following groups:

1. Atrophic states of the nasal structures, not accompanied by decomposition, whether or not associated with atrophy of the pharynx or larynx.

2. Atrophic states of the nasal structures with characteristic fetor, whether or not accompanied by atrophy of the pharynx or larynx.

3. Atrophic or dry conditions of the pharyngeal mucous membrane unaccompanied by atrophy within the nose.

For convenient designation these conditions will be called respectively, non-fetid atrophy, fetid atrophy and pharyngeal atrophy.

The relations of these atrophic states to sex are non-fetid atrophy, males 20, females 54; fetid atrophy, males 28, females 71 ; pharyngeal atrophy, males 12, females 15.

An Experimental Study of the Respiratory Functions of the Nares Boston Medical and Surgicai Journal of November 5 and 12, 1896. 